\title{
ENHANCING POWER ELECTRONIC DEVICES WITH WIDE BANDGAP SEMICONDUCTORS
}

\author{
BURAK OZPINECI \\ Oak Ridge National Laboratory \\ Oak Ridge, TN 37831-6472 USA \\ ozpinecib@ornl.gov \\ MADHU SUDHAN CHINTHAVALI \\ Oak Ridge Institute for Science and Education \\ Oak Ridge, TN 37831-0117 USA \\ chinthavalim@ornl.gov \\ LEON M. TOLBERT* \\ The University of Tennessee \\ Knoxville, TN 37996-2100 USA \\ tolbert@utk.edu
}

\begin{abstract}
Silicon carbide (SiC) unipolar devices have much higher breakdown voltages than silicon (Si) unipolar devices because of the ten times greater electric field strength of $\mathrm{SiC}$ compared with $\mathrm{Si}$. $4 \mathrm{H}-$ $\mathrm{SiC}$ unipolar devices have higher switching speeds due to the higher bulk mobility of $4 \mathrm{H}-\mathrm{SiC}$ compared to other polytypes. In this paper, four commercially available SiC Schottky diodes with different voltage and current ratings, VJFET, and MOSFET samples have been tested to characterize their performance at different temperatures ranging from $-50^{\circ} \mathrm{C}$ to $175^{\circ} \mathrm{C}$. Their forward characteristics and switching characteristics in this temperature range are presented. The characteristics of the SiC Schottky diodes are compared with those of a Si pn diode with comparable ratings.
\end{abstract}

Keywords: SiC; MOSFET; JFET; Schottky diode.

\section{Introduction}

With the increase in demand for more efficient, higher power, and higher temperature operation of power converters, design engineers face the challenge of increasing the efficiency and power density of converters. ${ }^{1,2}$ Development in power semiconductors is vital for achieving the design goals set by the industry. Si power devices have reached their theoretical limits in terms of higher temperature and higher power operation by virtue of the physical properties of the material. To overcome these limitations, research has focused on wide bandgap materials, such as silicon carbide ( $\mathrm{SiC}$ ), gallium nitride

\footnotetext{
" Leon M. Tolbert is also a part-time employee of Oak Ridge National Laboratory.
} 
(GaN), and diamond because of their superior material advantages such as large bandgap, high thermal conductivity, and high critical breakdown field strength.

Diamond is the ultimate material for power devices because of its more than ten-fold better electrical properties; however, the diamond manufacturing process is still in its infancy. ${ }^{3}$ Considering that $\mathrm{SiC}$ (which is produced at much lower temperature) has many material issues, it is expected that diamond will have more materials issues. Diamond power devices might be available in a 20-50 year time frame.

$\mathrm{GaN}$ and $\mathrm{SiC}$ power devices have similar performance improvements over Si power devices. GaN performs only slightly better than $\mathrm{SiC}$. Both $\mathrm{SiC}$ and $\mathrm{GaN}$ have processing issues that need to be solved before they can seriously challenge $\mathrm{Si}$ power devices; however, $\mathrm{SiC}$ is at a more technically advanced stage than $\mathrm{GaN}$. $\mathrm{SiC}$ is concluded to be the best suitable transition material for future power devices before high power diamond device technology matures. Therefore, the authors are focused on SiC power devices and their system level benefits.

Since $\mathrm{SiC}$ power devices have lower losses, $\mathrm{SiC}$-based power converters are more efficient. With the high temperature operation capability of $\mathrm{SiC}$, thermal management requirements are reduced; therefore, a smaller heatsink would be sufficient. In addition to this, since $\mathrm{SiC}$ power devices can be switched at higher frequencies, smaller passive components are required in power converters. Smaller heatsink and passive components result in higher power density power converters.

$\mathrm{SiC}$ unipolar devices such as Schottky diodes, VJFETs, and MOSFETs have much higher breakdown voltages compared with their Si counterparts, which makes them suitable for use in medium-voltage applications. At present, SiC Schottky diodes are the only commercially available $\mathrm{SiC}$ devices. The maximum ratings on these commercial devices are $1200 \mathrm{~V}$ and $20 \mathrm{~A}$. Some other $600 \mathrm{~V}$ prototype Schottky diodes with 100A rating are in the experimental stage and are expected to be commercially available in the near future.

$\mathrm{SiC}$ Schottky diodes are being used in several applications and have proved to increase the system efficiency compared with $\mathrm{Si}$ device performance. ${ }^{4}$ Significant reduction in weight and size of $\mathrm{SiC}$ power converters with an increase in the efficiency is projected. ${ }^{1,2}$ In the literature, the performance of SiC converters has been compared to traditional Si converters and was found to be better than Si power converters. ${ }^{5,6}$

The gate drive is an important aspect of the converter design which contributes to the device performance and hence the system. The SiC power switches listed above and some SiC VJFETs reported in ${ }^{7,8}$ were switched using gate drive circuits designed with discrete components. However, in the circuit design presented in this paper, a commercial gate drive IC chip IXDD414 is used which makes the gate drive operation more reliable. These gate drives can be applied to SiC MOSFETs and VJFETs by selecting different gate resistance values and modifying the output voltage polarity.

This paper presents the characteristics for several SiC diodes and power switches, and compares their performance. Some applications require that devices be able to handle extreme environments that include a wide range of operating temperature. In the following sections, the static and dynamic performances of some commercially available 
SiC Schottky diodes and experimental samples of SiC VJFETs and MOSFETs in a wide temperature range will be presented.

\section{SiC Schottky Diodes}

SiC Schottky diodes are majority carrier devices and are attractive for high frequency applications because they have lower switching losses compared to pn diodes. However, they have higher leakage currents, which affect the breakdown voltage rating of the devices. ${ }^{9}$ SiC Schottky diodes tested in this paper are S1 (1200 V, $\left.7.5 \mathrm{~A}\right), \mathrm{S} 2$ (600 V, 4A), S3 (600 V, $10 \mathrm{~A})$, and S4 (300 V, $10 \mathrm{~A})$.

\subsection{Static Characteristics}

The static characteristics of different SiC Schottky diodes at room temperature are shown in Fig. 1. The threshold voltages (or the knee voltages) and the on-state resistances are different for the diodes because of the differences in device dimensions for different voltage and current ratings. The threshold voltage also varies with the contact metal used in the Schottky diodes because of the variation in the Fermi level for different metal to semiconductor contacts. The static characteristics of one of the diodes $(\mathrm{S} 3,600 \mathrm{~V}, 10 \mathrm{~A})$ in a temperature range of $-50^{\circ} \mathrm{C}$ to $175^{\circ} \mathrm{C}$ are shown in Fig. 2. The static characteristics of the rest of the diodes can be found in ${ }^{10}$.

The approximate on-state voltage drop equation of the diode is given as

$$
V_{f}=V_{d}+I_{d} \cdot R_{d}
$$

where $V_{d}$ is the forward voltage drop and $R_{d}$ is the series resistance of the diode obtained from the piece-wise linear (PWL) model of the diode. The PWL model parameters were extracted from the experimental test data in Fig. 2. The variation in $V_{d}$ with temperature

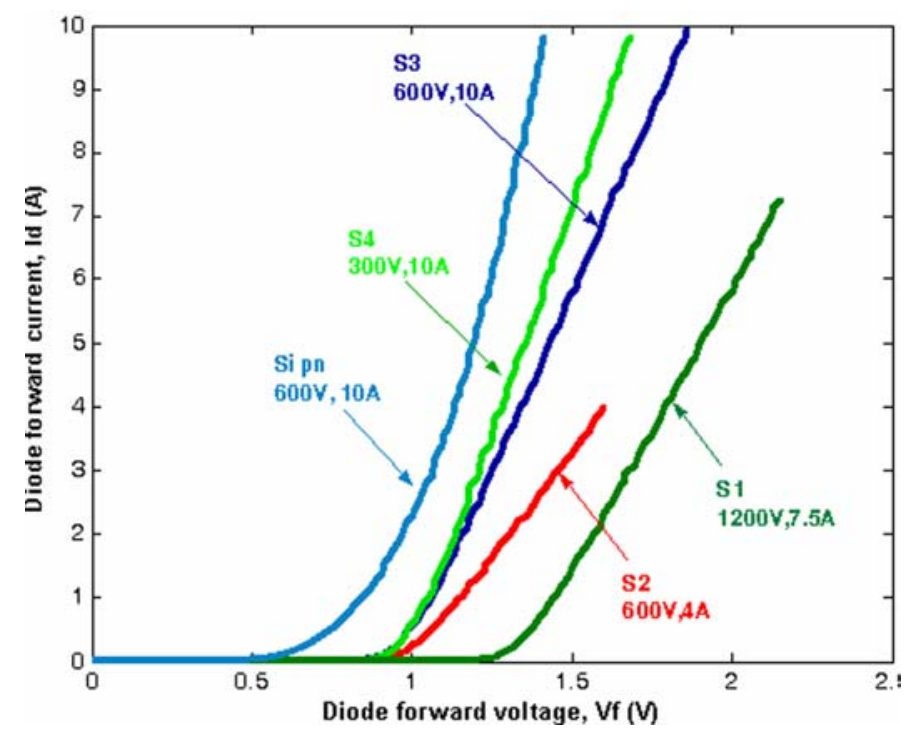

Fig. 1. $i-v$ characteristics of Si pn and SiC Schottky diodes at $27^{\circ} \mathrm{C}$. 


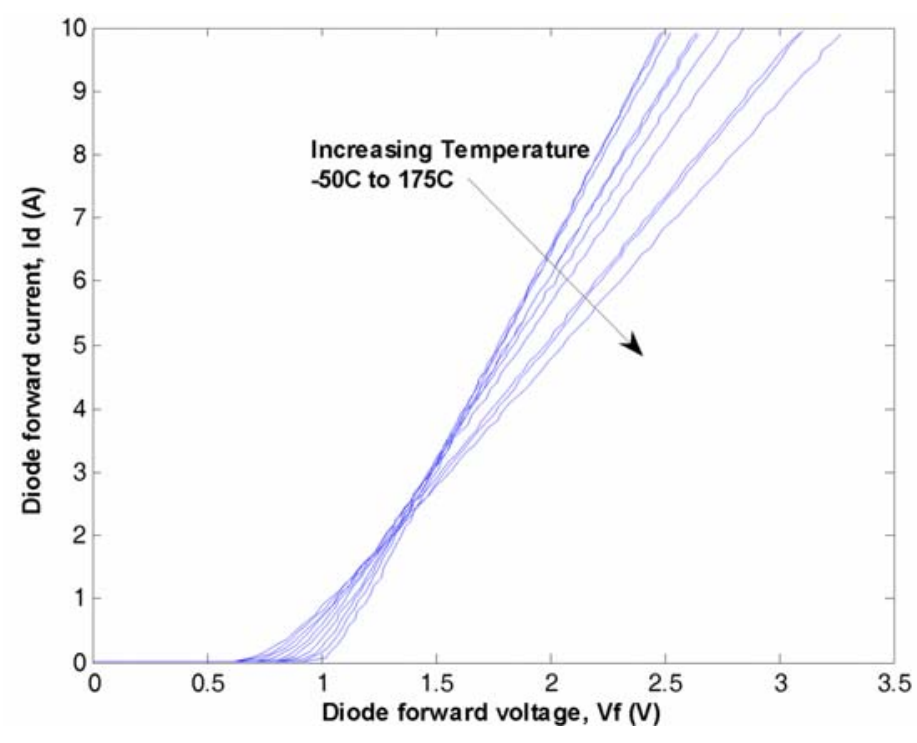

Fig. 2. $i-v$ characteristics of $\mathrm{S} 3(600 \mathrm{~V}, 10 \mathrm{~A})$ at different operating temperatures.

is plotted in Fig. 3 for all the diodes tested in this paper.

The on-state voltage drop of a Schottky diode is dependent on the barrier height and the on-state resistance, both of which vary with temperature. At lower current levels, as the temperature increases, the thermal energy of electrons increases, which causes lowering of the barrier height. A lower barrier height means a lower barrier potential and a lower forward voltage drop. ${ }^{11}$

At higher current levels the on-state voltage drop is mainly because of the series resistance of the diode, which is one of the critical parameters which determine the performance of the device. The dominant component of the diode series resistance is the

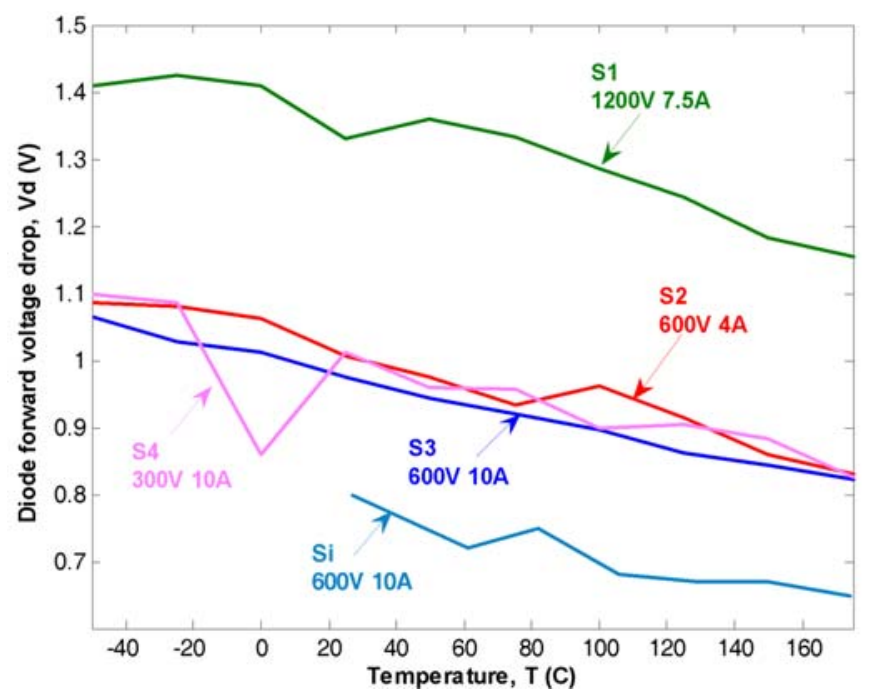

Fig. 3. $V_{d}$ for Si and SiC diodes at different operating temperatures. 


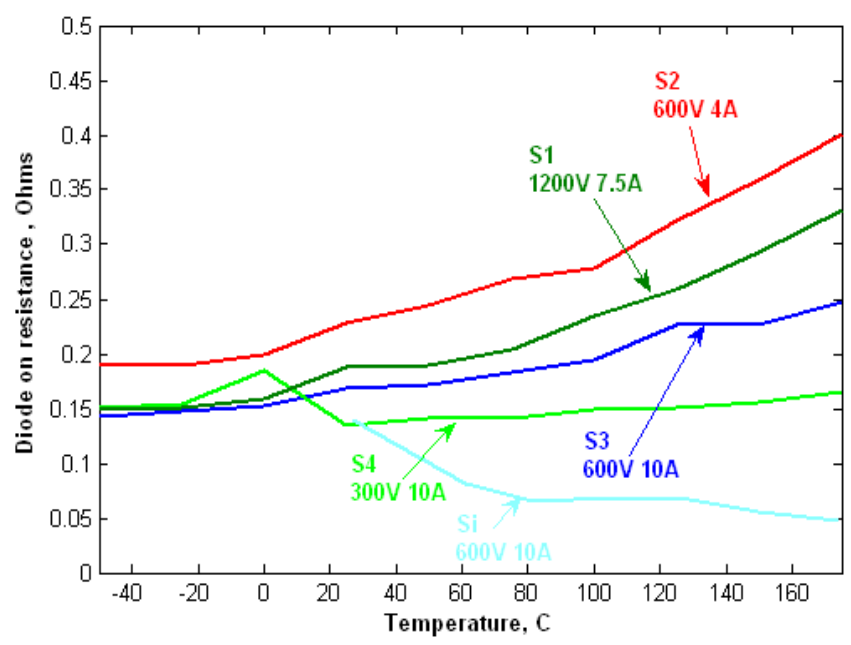

Fig. 4. $R_{d}$ for $\mathrm{Si}$ and $\mathrm{SiC}$ diodes at different operating temperatures.

specific on-resistance, $R_{o n, s p}$. The $R_{o n, s p}$ for majority carrier devices can be expressed as a function of breakdown voltage and critical electric field. ${ }^{8}$

$$
R_{o n, s p}=\frac{4 V_{B}^{2}}{\varepsilon\left(E_{c}\right)^{3} \mu_{n}}
$$

where $\varepsilon$ is the permittivity $(\mathrm{C} / \mathrm{V} \cdot \mathrm{cm}), V_{B}$ is the breakdown voltage, $E_{c}$ is the breakdown field $(\mathrm{V} / \mathrm{cm})$, and $\mu_{n}$ is the electron mobility $\left(\mathrm{cm}^{2} / \mathrm{V} \cdot \mathrm{s}\right)$.

$R_{o n, s p}$ increases with temperature because the mobility decreases at higher temperatures. Consequently, the diode series resistance increases with temperature and the device has positive temperature coefficient, which makes it easier to parallel these devices. The disadvantage, however, is that the diode conduction losses also increase with temperature.

The series resistance $R_{d}$ for the diodes is calculated from the slope of the $i$ - $v$ characteristics at high currents and is plotted for different temperatures as shown in Fig. 4. The series resistance of each diode is unique because of the differences in blocking voltages and the die area. To withstand high breakdown voltages, the blocking layer thickness must be increased, and doping concentrations must be reduced. This results in increased series resistance of the diode. Hence, device S1 rated at $1200 \mathrm{~V}, 7.5 \mathrm{~A}$ has a higher series resistance compared to S3 $(600 \mathrm{~V})$ and S4 $(300 \mathrm{~V})$. The resistance also varies with the area of the device. It is evident from Fig. 4 that S2 and S3 with the same voltage and different current ratings have different series resistances.

\subsection{Dynamic Characteristics}

A buck chopper with an inductive load is built to evaluate the switching characteristics of the diodes. A Si IGBT is used as the main switch and is switched at $20 \mathrm{kHz}$ with a $25 \%$ duty ratio. 


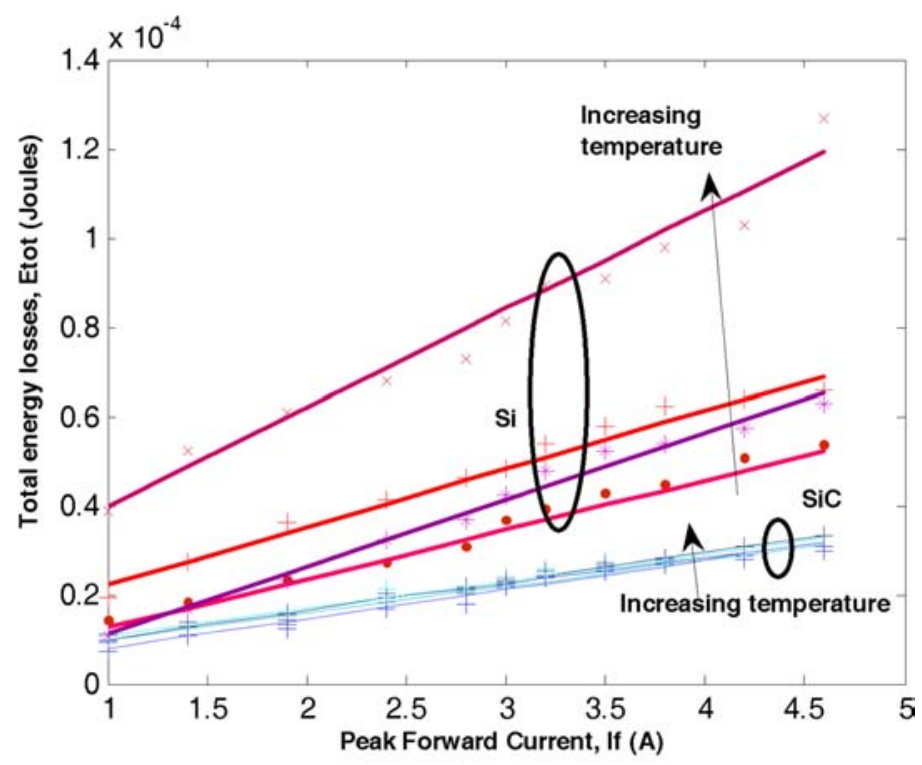

Fig. 5. Total energy losses with respect to forward current at different operating temperatures.

The energy losses for various forward peak currents and different temperatures are shown for the Si diode and $\mathrm{SiC}$ diode $\mathrm{S} 4$ in Fig. 5. The switching loss for the Si diode increases with temperature and forward current while the switching loss for the SiC diode S4 is almost independent of the change in temperature and varies slightly with increasing forward current.

The reverse recovery current of a diode is dependent on charge stored in the drift region. Schottky diodes have no stored charge because they are majority carrier devices and thus have no reverse recovery. However, oscillations due to parasitic internal pn diodes and capacitances look like reverse recovery phenomena. Reduced reverse recovery of Schottky diodes makes it possible to reduce the size of the snubbers. Low reverse recovery and snubber losses increase the efficiency of the power converters.

\section{SiC FET Devices}

FET devices are majority carrier devices and are preferred to minority carrier devices in power converters; however, Si FET devices, like Si Schottky diodes, can only be used in low-voltage $(<300 \mathrm{~V})$ applications. Even the first experimental SiC FET devices have blocking voltages over $1000 \mathrm{~V}$. It is expected that in the near future, SiC FET devices will dominate Si minority carrier devices in the medium-voltage $(<3000 \mathrm{~V})$ applications. 


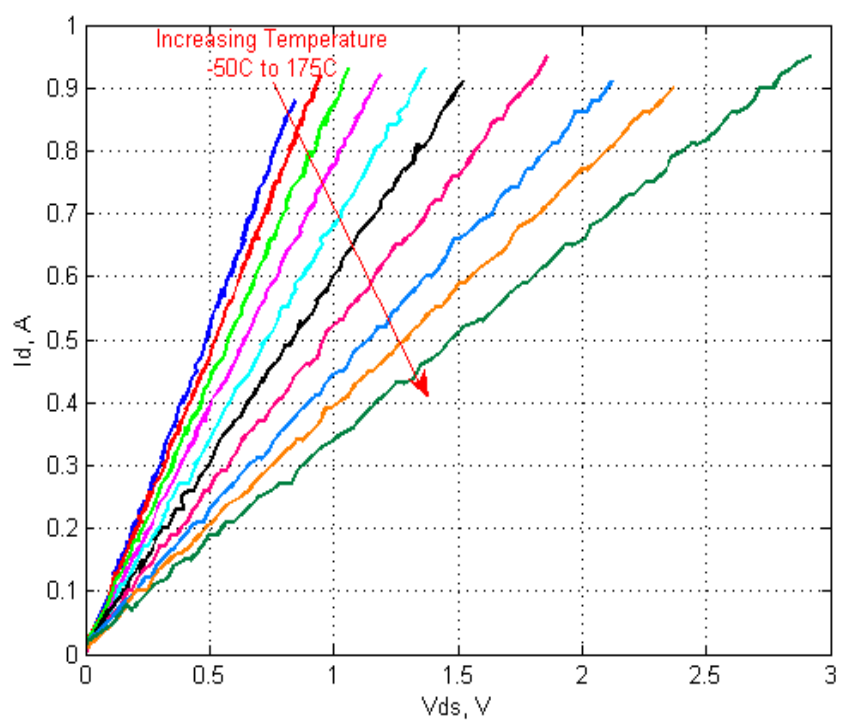

Fig. 6. $i-v$ characteristics of SiC VJFET at different temperatures.

\subsection{Static Characteristics}

\subsubsection{SiC Vertical JFET (VJFET)}

A JFET is a unipolar device and has several advantages compared with MOSFET devices. A JFET has a low voltage drop and a higher switching speed, and a JFET is free from the gate oxide interface problems unlike the MOSFET. ${ }^{12}$ A SiC VJFET is typically a normally-on device and conducts even though there is no gate voltage applied. A gate voltage has to be applied for it to stop conduction. A normally-on device is not desirable for power electronics since it requires additional protection circuitry to prevent a dc bus short if the gate signals fail. This normally-on feature also demands special gate drive designs increasing the complexity of design.

A Si JFET is usually an electronics device and is not classified as a power electronics device. The SiC VJFET, however, can be used in high-voltage, high-power applications unlike a Si JFET because of its vertical structure and the intrinsic properties of SiC. A normally-on SiC VJFET rated at $1200 \mathrm{~V}$ and $2 \mathrm{~A}$ was tested to study the high temperature behavior of the device. The forward characteristics of this device at different temperatures are shown in Fig. 6. The on-resistance of the VJFET increases from $0.36 \Omega$ at $-50^{\circ} \mathrm{C}$ to $1.4 \Omega$ at $175^{\circ} \mathrm{C}$ as shown in Fig. 7. The values of the on-resistance are high; however, this device is a low current rated device and it is one of the first of its kind. It is expected that as the technology matures lower on-resistance will be possible.

As seen in Figs. 6 and 7, SiC VJFETs have positive temperature coefficients, which means that, like SiC Schottky diodes, their conduction losses will be higher at higher temperatures. Positive temperature coefficient makes it easier to parallel these devices and reduce the overall on-resistance. 


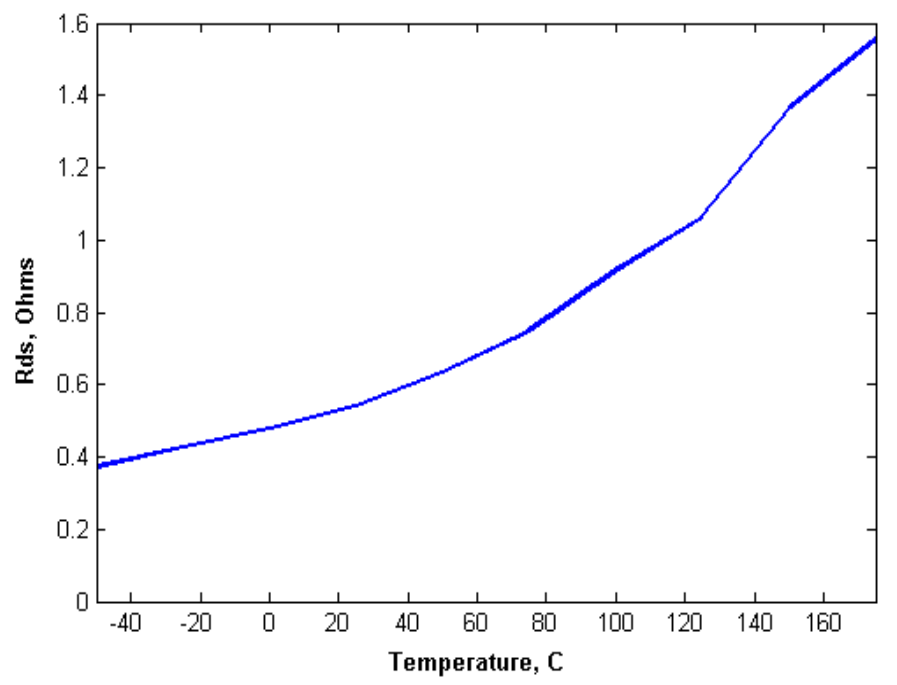

Fig. 7. On-resistance of SiC VJFET at different temperatures.

The transfer characteristics of different SiC VJFET samples are shown in Fig. 8. The negative gate pinch-off voltage required to turn-off the device is higher than that required for $\mathrm{Si}$ devices and varies from sample to sample. This variation is attributed to the fact that these devices are experimental samples and is not expected to exist in future commercial SiC VJFETs. Since the pinch-off voltage determines the voltage requirement of the gate drive circuit, the gate driver needs to be designed considering this variation and higher voltage requirement in order to switch any of these devices.

\subsubsection{SiC MOSFET}

A MOSFET is a unipolar device and is normally off. A SiC MOSFET can block voltages

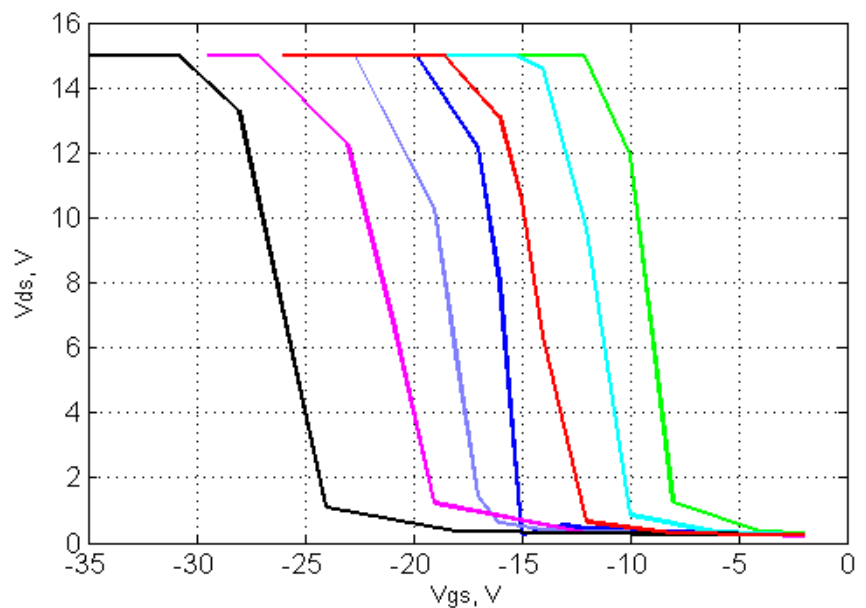

Fig. 8. Transfer characteristics of several SiC VJFET samples. 


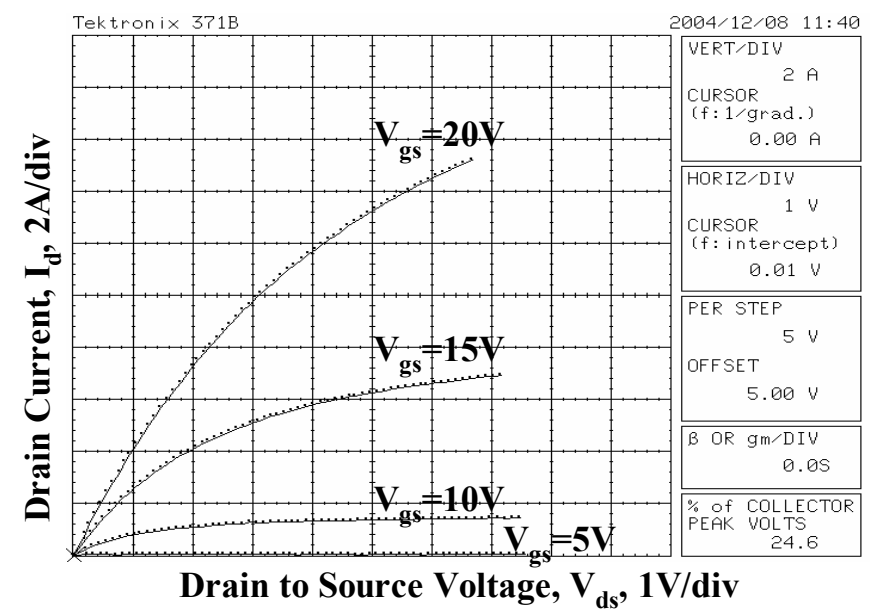

Fig. 9. Forward characteristics of SiC MOSFET at room temperature.

more than $3 \mathrm{kV}$ unlike a Si MOSFET, which can typically block up to $300 \mathrm{~V}$, because of the high electric breakdown field strength of $\mathrm{SiC}$.

The forward characteristics of a $1.2 \mathrm{kV}, 15 \mathrm{~A} \mathrm{SiC} \mathrm{MOSFET} \mathrm{at} \mathrm{room} \mathrm{temperature} \mathrm{are}$ shown in Fig. 9. The gate voltage is varied from $0 \mathrm{~V}$ to $20 \mathrm{~V}$ with increments of $5 \mathrm{~V}$. For $V_{g s}=20 \mathrm{~V}$, there is a $6.7 \mathrm{~V}$ drain-to-source voltage drop that corresponds to $15 \mathrm{~A}$ drain current. Note that it would be more reasonable to operate this device at $5 \mathrm{~A}$ with $V_{g s}=20 \mathrm{~V}$ because of a low voltage drop of $1.5 \mathrm{~V}$. The forward characteristics of the same $\mathrm{SiC}$ MOSFET are shown in Fig. 10 for a temperature range of $-50^{\circ} \mathrm{C}$ to $175^{\circ} \mathrm{C}$. Note that the device's response to temperature increase changes at $50^{\circ} \mathrm{C}$. To get a better understanding of this phenomenon, the on-state resistances of the device are calculated from the slopes of the different curves and are plotted with respect to temperature in Fig. 11. It is interesting to note that this device has a negative temperature coefficient up to $50^{\circ} \mathrm{C}$ and positive temperature coefficient above that. MOSFETs are majority carrier devices and are expected to be devices with positive temperature coefficients. For better insight, consider the components of the on-resistance of a MOSFET, which can be expressed as the sum of several different resistances because of different regions of the MOSFET structure:

$$
R_{\text {on }}=R_{\text {cont }}+R_{\text {sub }}+R_{c h}+R_{a c c}+R_{j f e t}+R_{d}
$$

where $R_{\text {cont }}$ is contact resistance, $R_{\text {sub }}$ is substrate resistance, $R_{c h}$ is channel resistance, $R_{a c c}$ is accumulation layer resistance, $R_{\text {jfet }}$ is resistance of JFET like region, and $R_{d}$ is resistance of the drift region. ${ }^{13}$

At lower temperatures, the contribution of the channel resistance to the total on-state resistance is dominant. ${ }^{14}$ The channel mobility increases with temperature because of the interface traps being closer to the conduction band. ${ }^{14,15}$ The channel resistance decreases with an increase in temperature because of the increase in the channel mobility with temperature. Therefore, at low temperatures, the MOSFET on-resistance decreases, too. 


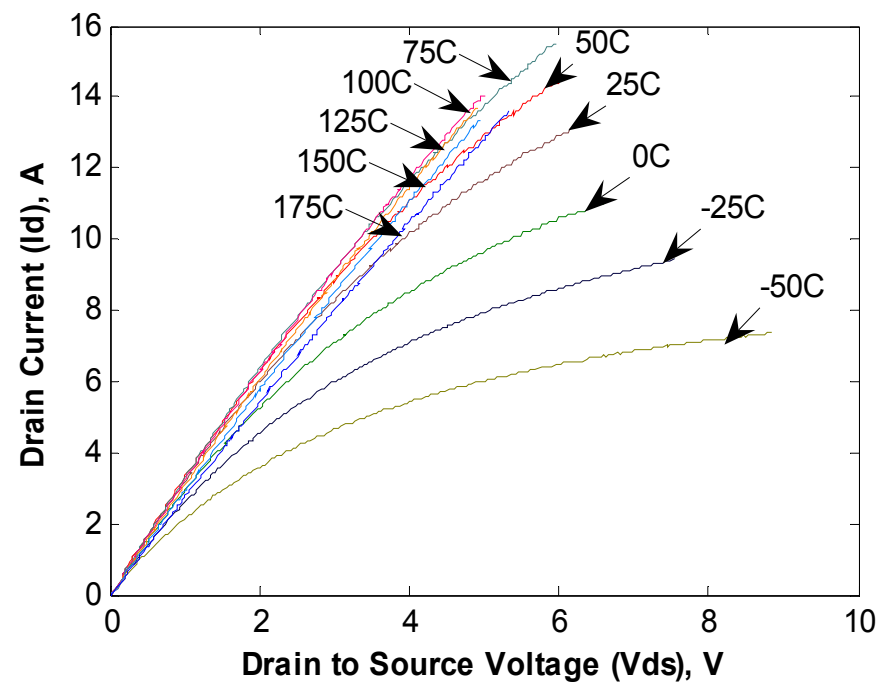

Fig. 10. Forward characteristics of SiC MOSFET at different temperatures.

Above a certain temperature value, $R_{c h}$ is not dominant and because of the other dominant on-resistance components, the MOSFET overall on-resistance increases.

The SiC MOSFET gate threshold voltage is measured from the transfer characteristics shown in Fig. 12. The gate threshold voltage decreases with increasing temperature. Fig. 13 shows the change in threshold voltage from $10.7 \mathrm{~V}$ at $-50^{\circ} \mathrm{C}$ to $2.8 \mathrm{~V}$ at $175^{\circ} \mathrm{C}$. This change is due to the trapped charge in the $\mathrm{SiO}_{2}$ as well as the impurities at the $\mathrm{SiO}_{2}$ interface. These trapped charges become active at high temperatures, which results in a Fermi level shift towards the bandgap causing the drain current to flow at low threshold voltages. In other words, less gate voltage is required at high temperatures for the same drain current to flow through the device.

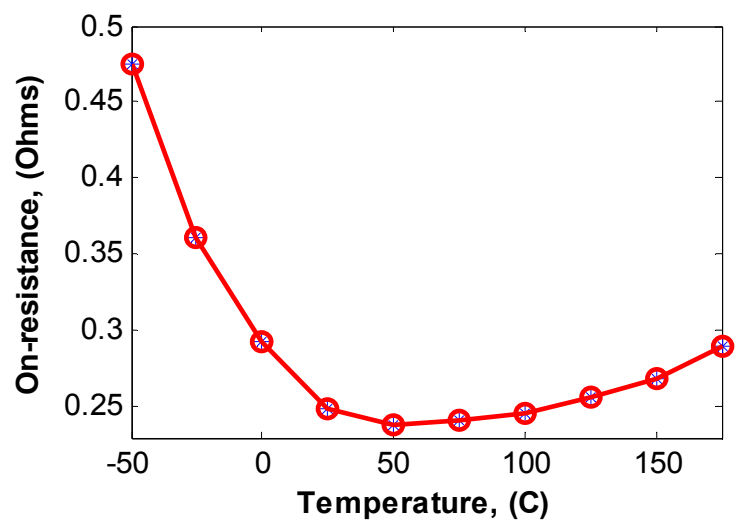

Fig. 11. On-resistance of SiC MOSFET at different temperatures. 


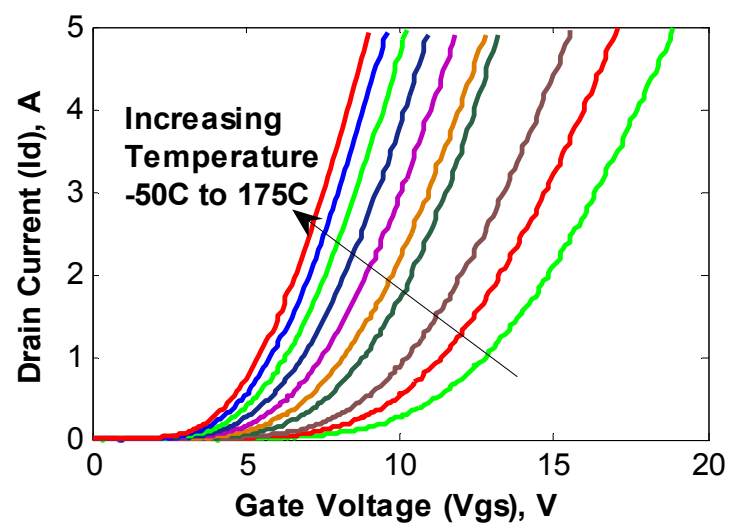

Fig. 12. Transfer characteristics of SiC MOSFET at different temperatures.

\subsection{Gate Drive Requirements}

SiC FET switches can be operated at higher switching frequencies and higher temperatures; therefore, they have different gate drive requirements compared to traditional $\mathrm{Si}$ power switches. The switching performance of the FET devices is determined by charging and discharging of the parasitic capacitances across the three terminals, input capacitance, reverse transfer capacitance, and output capacitance. These capacitances are proportional to the area of the device. ${ }^{6}$ Since $\mathrm{SiC}$ devices have smaller areas than comparable Si devices, even for high blocking voltages the capacitances are reduced. This enables devices to operate at higher switching speeds. A comparison of capacitance values for a SiC MOSFET and Si power switches is reported in ${ }^{16}$.

One of the important parameters in gate drive design is the stray capacitance between the gate and the other terminals. Total input capacitance of the VJFET, $C_{i s s}$, determines

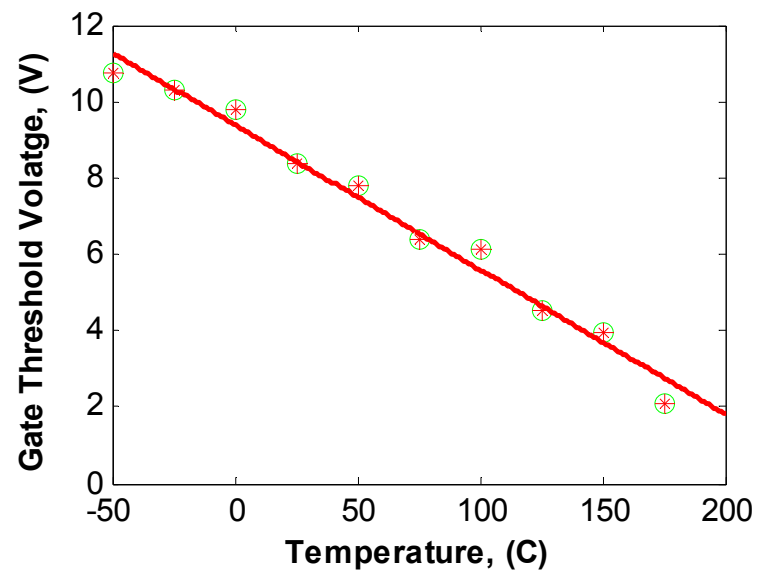

Fig. 13. Gate threshold voltage of SiC MOSFET at different temperatures. 
the current required by the gate and the rate at which the applied gate voltage is built across the gate and source terminals. Therefore, the gate drive circuit is required to have the capability of providing peak currents to be able to charge the input capacitance quickly. The peak gate current is limited by the series resistance between the gate and the gate driver output. The gate series resistance decreases the ringing effect due to the internal impedance of the device. However, increasing the gate resistance value results in slower turn-on times.

As mentioned earlier, in the literature, there are several gate drive circuit designs for SiC VJFETs using transistors and other discrete devices. ${ }^{7,8}$ In this paper, a commercial gate driver IC chip IXDD414 is used to drive SiC FETs for a more reliable and quick gate driver design. Note that this gate driver IC can be used with most of the SiC JFETs whose transfer characteristics are shown in Fig. 8 as well as the SiC MOSFET samples. The gate driver can be redesigned to be used as a SiC MOSFET gate drive by replacing the series gate resistor and reversing the output voltage polarity. The peak gate currents and gate voltage waveforms driving a SiC VJFET and a SiC MOSFET are shown in Fig. 14.

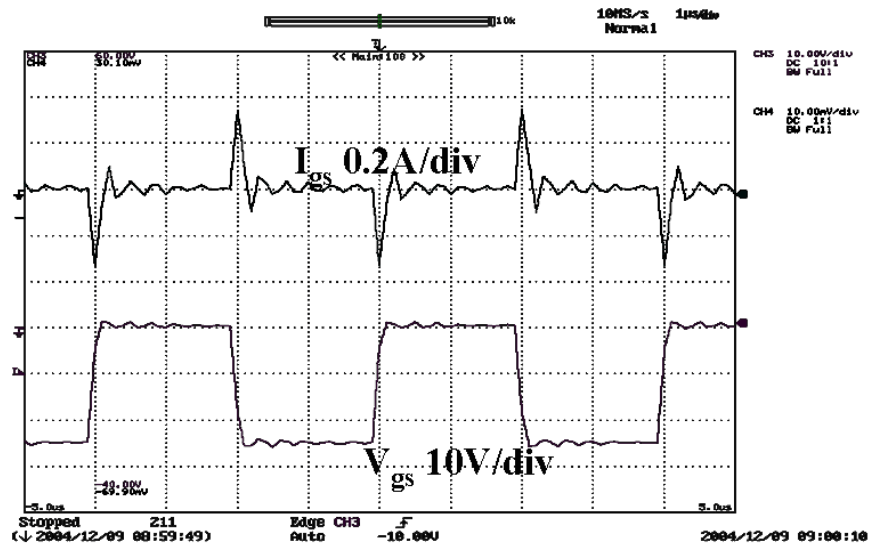

(a)

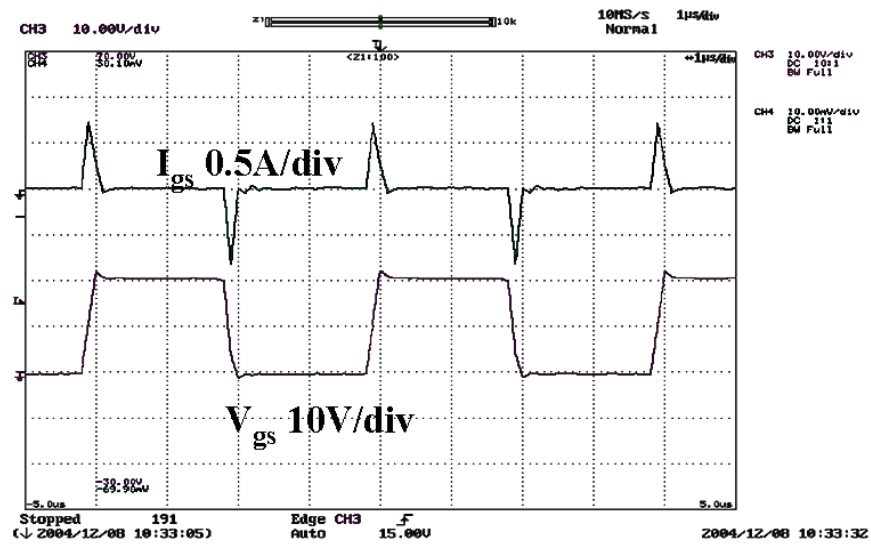

(b)

Fig. 14. The peak gate currents and gate voltage waveforms with (a) SiC VJFET, (b) SiC MOSFET. 


\subsection{Dynamic Characteristics}

The gate drive circuit discussed in the previous section was used to determine the dynamic characteristics of the SiC MOSFET and VJFET.

$\mathrm{SiC}$ VJFETs are normally-on devices and they can only be turned off by applying a negative voltage that is higher than what a typical Si power device requires. Based on the transfer characteristics (Fig. 8), the gate drive was designed for a voltage of $-25 \mathrm{~V}$ since the it would be enough to turn off most of the samples tested.

When SiC VJFETs are operated at high frequencies, they need high peak gate currents to charge and discharge the gate capacitances faster. A $250 \mathrm{kHz}$ operation was achieved with a series gate resistance of $5.4 \Omega$ and a peak gate current of $0.38 \mathrm{~A}$.

The gate voltage and the switching waveforms of the VJFET are shown in Fig. 15. The device has a turn-off delay $t_{d, \text { off }}$ of $40 \mathrm{~ns}$, fall time $t_{f}$ of $80 \mathrm{~ns}$, turn-on delay $t_{d, o n}$ of $20 \mathrm{~ns}$, and rise time $t_{r}$ of $100 \mathrm{~ns}$.

The gate drive voltage for the MOSFET was selected to be $20 \mathrm{~V}$ as determined from the forward characteristics to obtain the optimum performance. A $250 \mathrm{kHz}$ operation was achieved with a series gate resistance of $7.2 \Omega$ and a peak gate current of $0.6 \mathrm{~A}$. The gate

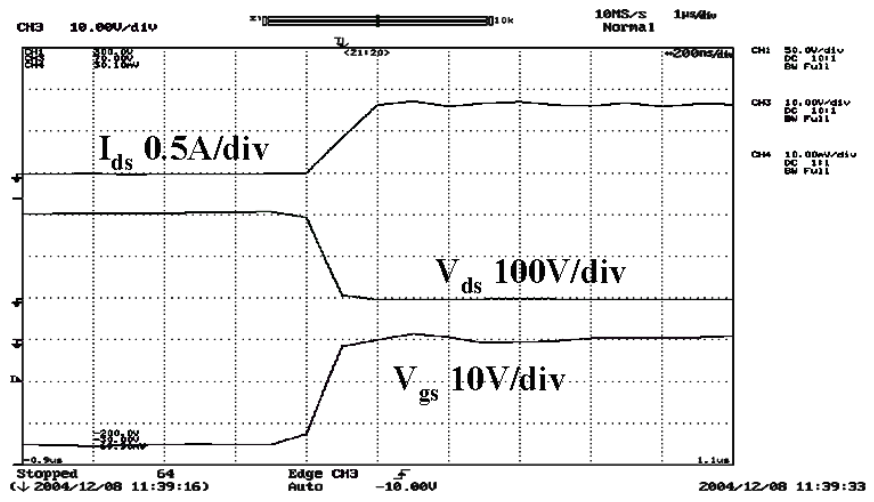

(a)

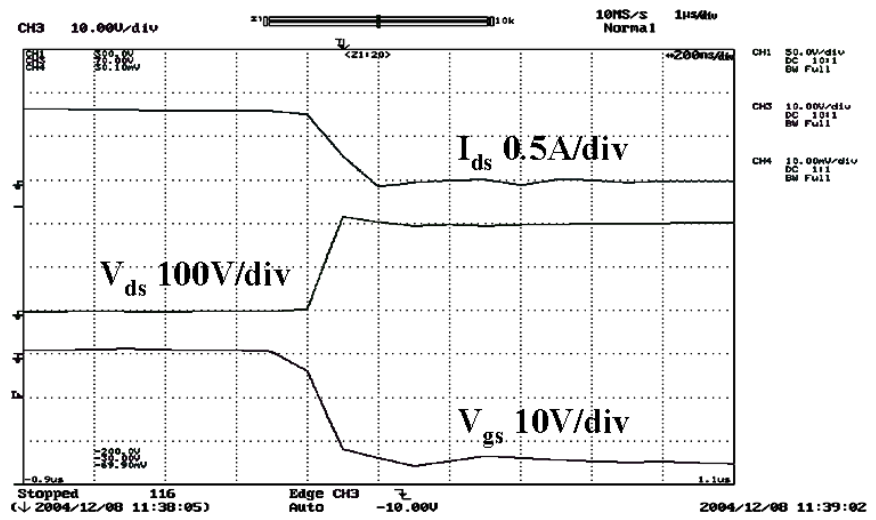

(b)

Fig. 15. The gate and switching waveforms of the SiC VJFET. 
and switching waveforms for a SiC MOSFET are shown in Fig. 16. The device has a turn-off delay $t_{d, \text { off }}$ of $40 \mathrm{~ns}$, fall time $t_{f}$ of $100 \mathrm{~ns}$, turn-on delay $t_{d, o n}$ of $20 \mathrm{~ns}$, and rise time $t_{r}$ of $100 \mathrm{~ns}$.

The turn on and turn off energy losses for both the SiC MOSFET and VJFET were calculated by integrating the instantaneous power over the turn on $\left(t_{o n}\right)$ and turn off times $\left(t_{o f f}\right)$.

The energy losses calculated for the SiC MOSFET and JFET at different temperatures, for a $5 \mathrm{kHz}, 50 \%$ duty cycle, $100 \mathrm{~V}, 0.8 \mathrm{~A}$ operation are shown in Fig. 17. Note that the switching losses do not change much with temperature.

\section{Conclusions}

The static and dynamic performances of some SiC Schottky diodes, SiC MOSFETs, and $\mathrm{SiC}$ VJFETs were characterized. It was observed that SiC Schottky diodes and VJFETs have positive temperature coefficient behavior. However, SiC MOSFETs display

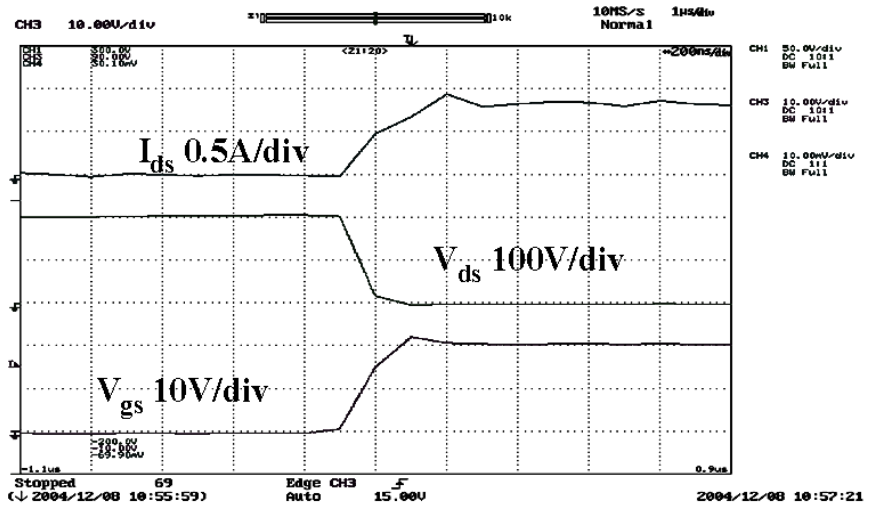

(a)

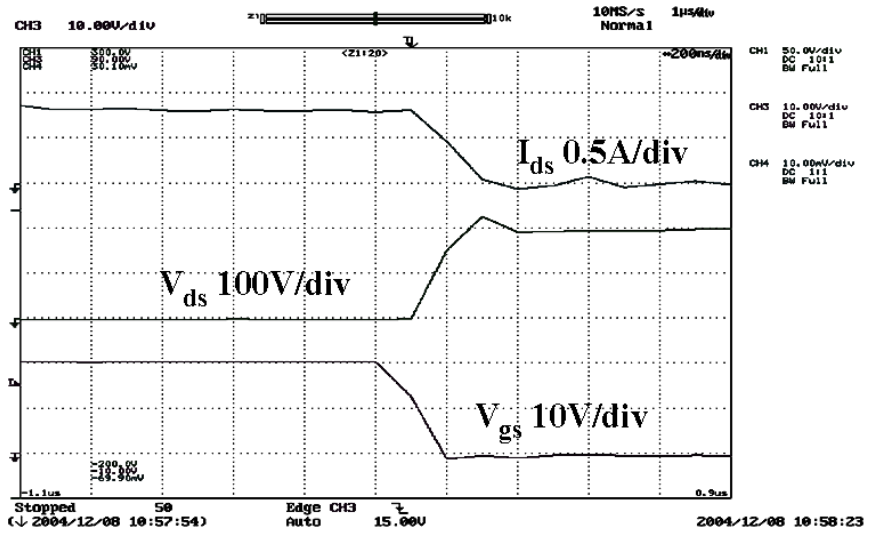

(b)

Fig. 16. The gate and switching waveforms of the SiC MOSFET. 


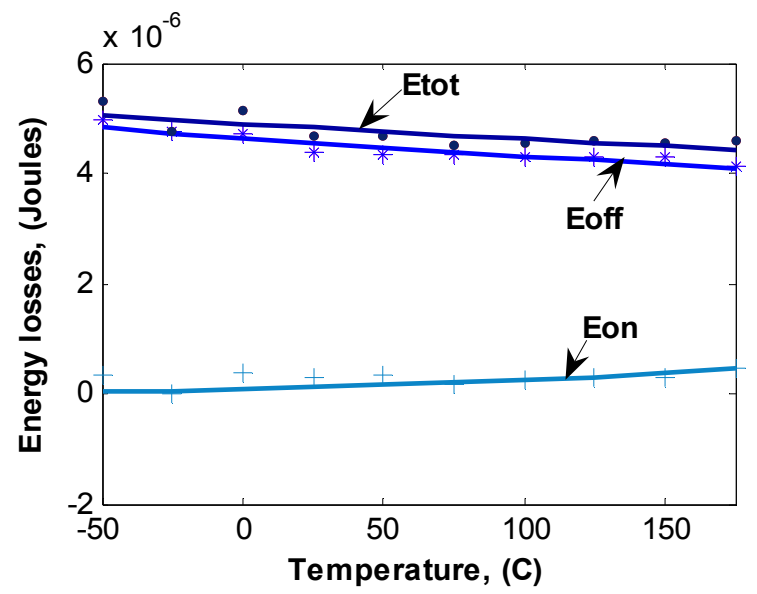

(a)

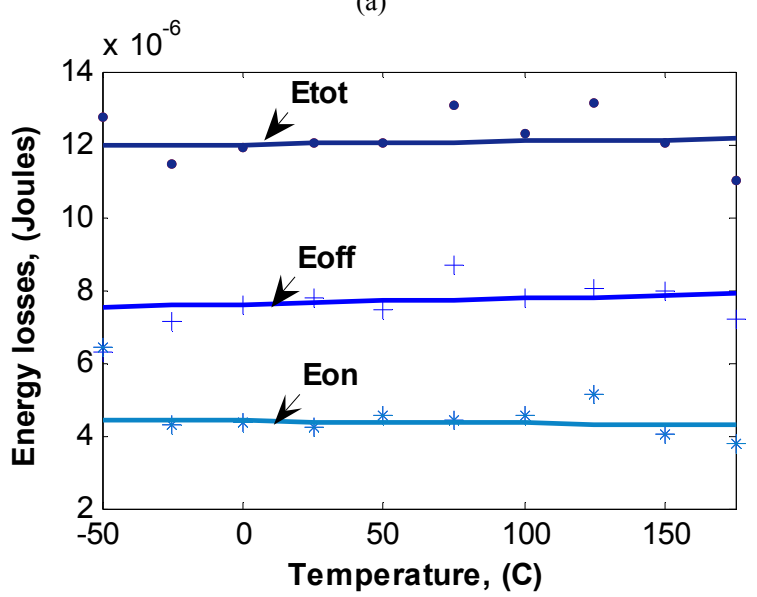

(b)

Fig. 17. Energy loss plots for (a) VJFET, (b) MOSFET.

negative temperature behavior for temperatures below $50^{\circ} \mathrm{C}$ and positive temperature coefficient above this value. This is because of the interface trap defects in the $\mathrm{SiC}$ MOSFET which will eventually be eliminated with maturing $\mathrm{SiC}$ manufacturing technology.

$\mathrm{SiC}$ Schottky diodes showed excellent reverse recovery characteristics compared to $\mathrm{Si}$ pn diodes. Even though SiC Schottky diodes have higher on-resistances, their near perfect switching characteristics make them perform better than their Si counterparts. Thus, replacing $\mathrm{Si}$ devices with comparable $\mathrm{SiC}$ Schottky diodes will improve the performance of the power switches in a power converter by reducing the switching stress on the switches and decreasing the overall losses.

In this paper, as opposed to the others in the literature, a commercial gate driver chip was used in the design of a gate driver for both SiC MOSFETs and VJFETs. 
The switching losses were almost constant for a wide temperature range for all the $\mathrm{SiC}$ unipolar devices reported in this paper.

This shows that $\mathrm{SiC}$ unipolar devices are well suited for high-frequency, hightemperature, and high-power applications. Also, hard switching circuits at higher power levels and higher frequencies can be realized using $\mathrm{SiC}$ devices due to the excellent switching characteristics. With further improvements in current ratings, SiC MOSFETs can replace IGBTs. They are preferred over VJFETs because of SiC MOSFET's normally-off feature. However, the gate oxide reliability still remains an issue for $\mathrm{SiC}$ MOSFETs.

\section{Acknowledgments}

Prepared by the Oak Ridge National Laboratory, Oak Ridge, Tennessee 37831, managed by UT-Battelle for the U.S. Department of Energy under contract DE-AC05-00OR22725.

The submitted manuscript has been authored by a contractor of the U.S. Government under Contract No. DE-AC05-00OR22725. Accordingly, the U.S. Government retains a non-exclusive, royalty-free license to publish from the contribution, or allow others to do so, for U.S. Government purposes.

\section{References}

1. B. Ozpineci, L. M. Tolbert, S. K. Islam, F. Z. Peng, "Testing, characterization, and modeling of $\mathrm{SiC}$ diodes for transportation applications," IEEE Power Electronics Specialists Conference, June 23-27, 2002, Cairns, Australia, pp. 1673-1678.

2. L. M. Tolbert, B. Ozpineci, S. K. Islam F. Z. Peng, "Impact of SiC power electronic devices for hybrid electric vehicles," SAE 2002 Transactions Journal of Passenger Cars - Electronic and Electrical Systems, 2003, ISBN 0-7680-1291-0, pp. 765-771.

3. B. Ozpineci, L. M. Tolbert, "Comparison of Wide Bandgap Semiconductors for Power Electronics Applications," A technical report for the U.S. Department of Energy, ORNL/TM2003-257, November 2003.

4. S. Hodge, "SiC Schottky diodes in power factor correction," Power Electronics Technology, August 2004, pp. $14-18$.

5. H. R. Chang, E. Hanna, A. V. Radun, "Demonstration of silicon carbide (SiC) - based motor drive," Conference of the IEEE Industrial Electronics Society, vol. 2, 2-6 November 2003, pp. $1116-1121$.

6. A. M. Abou-Alfotouh, A. V.Radun, H. R. Chang, C. Winerhalter, "A $1 \mathrm{MHz}$ hard-switched silicon carbide DC/DC converter," IEEE Applied Power Electronics Conference, vol. 1, 9-13 February 2003, pp. $132-138$.

7. K. Mino, K. S. Herold, J. W. Kolar, "A gate drive circuit for silicon carbide JFET," Conference of the IEEE Industrial Electronics Society, vol. 2, 2-6 November 2003, pp. 1162 1166.

8. M. L. Heldwein, J. W. Kolar, "A novel SiC J-FET gate drive circuit for sparse matrix converter applications," IEEE Applied Power Electronics Conference, vol. 1, 22-26 February 2004, pp. $116-121$.

9. M. Bhatnagar, P. K. McLarty, B. J. Baliga, "Silicon carbide high voltage (400V) Schottky barrier diodes," IEEE Electron Device Letters, vol. 13, no. 10, October 1992, pp. 501-503. 
10. M. Chinthavali, B. Ozpineci, L. M. Tolbert, "High temperature characterization of SiC power electronic devices" IEEE Workshop on Power Electronics in Transportation, October 21-22, 2002, pp. 43-47.

11. A. R. Hefner, R. Singh, J. Lai, D. W. Berning, S. Bouche, C. Chapuy, "SiC power diodes provide breakthrough performance for a wide range of applications," IEEE Transactions on Power Electronics, vol. 16, no. 2, March 2001, pp. 273-280.

12. B. Allebrand, H. Nee, "On the possibility to use SiC JFETs in power electronic circuits," European Conference on Power Electronics and Applications, Austria, 2001.

13. M. Ruff, H. Mitlehner, R. Helbig, "SiC devices: Physics and numerical simulation," IEEE Transactions on Electron Devices, vol. 41, no. 6, June 1994, pp. 1040-1054.

14. D. Peters, H. Mitlehner, R. Elpelt, R. Schorner, D. Stephani, "State of the art technological challenges of $\mathrm{SiC}$ power MOSFETs designed for high blocking voltages," European Conference on Power Electronics and Applications, 2-4 September 2003, Toulouse, France.

15. S. H. Ryu, A. Agarwal, J. Richmond, J. Palmour, N. Saks, J. Williams, "10A, 2.4kV power DIMOSFETs in 4H-SiC," IEEE Electron Device Letters, vol. 23, no. 6, June 2002, pp. 321323.

16. S. H. Ryu, S. Krishnaswami, M. Das, J. Richmond, A. Agarwal, J. Palmour, J. Scofield, “4HSiC DMOSFETs for high speed switching applications," 5th European Conference on Silicon Carbide Related Materials, August 31- September 4, 2004 